\title{
SELECTING DIFFERENT INDUSTRIAL COMPETITORS INFLUENCE THE RISK LEVEL OF VIETNAM TELECOMMUNICATION AND EDUCATION COMPANIES
}

\author{
Dinh Tran Ngoc Huy ${ }^{1}$
}

\begin{abstract}
This research shows marketing factors such as business competitors could affect business market risk, from a quantitative point of view. Using a two (2) factors model, this research paper estimates the impacts of not only the size of firms' competitors, but also leverage in the telecommunication and education industry, on the market risk of 18 listed companies in this category.

This paper founds out that the risk dispersion level in this sample study could be minimized in case the competitor size is approximately the same (measured by equity beta var of 0,283) and leverage down to $20 \%$.

Beside, the emprical research findings show us that when financial leverage increases up to $30 \%$, max asset beta value decreases from 0,393 to 0,386 in case the size of competitor doubles or slightly smaller.

Last but not least, this paper illustrates calculated results that might give proper recommendations to relevant governments and institutions in re-evaluating their policies during and after the financial crisis 2007-2011.
\end{abstract}

Keywords: risk management, competitive firm size, market risk, asset and equity beta, education and telecommunication industry

JEL Classification : M00, G3, M3

\section{Introduction}

In marketing and business, choosing competitors might affect business strategies, esp., during the crisis period 2007-2009 in which telecommunication and education firms experience many risks, although Viet Nam telecommunication and education industry is considered as one of active economic sectors, which has some positive effects for the economy.

This paper is organized as follow. The research issues and literature review will be covered in next sessions 2 and 3, for a short summary. Then, methodology and conceptual theories are introduced in session 4 and 5. Session 6 describes the data in

\footnotetext{
${ }^{1} M B A, P h D$ candidate, Banking University, HCMC - GSIM, International University of Japan, Japan, dtnhuy2010@gmail.com
} 
empirical analysis. Session 7 presents empirical results and findings. Next, session 8 covers the analytical results. Then, session 9 presents analysis of industry. Lastly, session 10 will conclude with some policy suggestions. This paper also supports readers with references, exhibits and relevant web sources.

\section{Research Issues}

For the estimating of impacts of the selection of different industrial competitors on the risk measured by beta for listed telecommunication and education companies in Viet Nam stock exchange, research issues will be mentioned as following:

Issue 1: Whether the selection of different competitors makes the risk level of telecommunication and education industry firms under the different changing scenarios of leverage increase or decrease so much.

Issue 2: Whether the selection of doubling size competitor makes the dispersion of beta values become large in the different changing scenarios of leverage in this industry.

\section{Literature review}

Goldsmith (1969), Mc Kinnon (1973) and Shaw (1973) pointed a large and active theoretical and empirical literature has related financial development to the economic growth process.

Gosh and Morita (2007) pointed although collaboration between competitors reduces the distinctiveness between their products, it increases the distinctiveness between their products and the non-collaborators' product. Simkovic (2011) found out competition between mortgage securitizers led to a race to the bottom on mortgage underwriting standards that ended in the late 2000s financial crisis. Li and Netessin (2011) stated competition has become an important theme in the operations management literature and, according to recent theoretical and empirical work, the key finding is that firms tend to overstock or overproduce under competition.

Last but not least, Ana and John (2013) Binomial Leverage - Volatility theorem provides a precise link between leverage and volatility. Chen et all (2013) supports suspicions that over-reliance on short-term funding and insufficient collateral compounded the effects of dangerously high leverage and resulted in undercapitalization and excessive risk exposure for Lehman Brothers.

\section{Conceptual theories}

Industrial competitor theories

A firm can face many kinds of risk: operational risk or financial risk. These risks lead to lower production output and create opportunities for competitors to enter and expand their market share.

Business risks are affected by some variables including: sales volume, unit price and input costs. And competiton can affect these variables; so, it has impacts on business risks. 


\section{Methodology}

In this research, analytical research method is used, philosophical method is used and specially, scenario analysis method is used. Analytical data is from the situation of listed commercial electric industry firms in VN stock exchange and applied current tax rate is $25 \%$. And the below table 1 shows us three different cases of selecting competitors.

Finally, we use the results to suggest policy for both these enterprises, relevant organizations and government.

Table 1 - Analyzing market risk under three (3) scenarios of changing competitors (Made by Author)

\begin{tabular}{|c|c|c|c|c|}
\hline $\begin{array}{l}\text { Order } \\
\text { No. }\end{array}$ & $\begin{array}{l}\text { Company Stock } \\
\text { code }\end{array}$ & $\begin{array}{l}\text { Competitor size } \\
\text { as current }\end{array}$ & $\begin{array}{l}\text { Competitor size } \\
\text { slightly smaller }\end{array}$ & $\begin{array}{l}\text { Competitor size } \\
\text { double }\end{array}$ \\
\hline 1 & ECI & & & \\
\hline 2 & INN & & & \\
\hline 3 & PTP & & & \\
\hline 4 & DHI & & & \\
\hline 5 & IHK & $\begin{array}{l}\text { TPH as } \\
\text { comparable }\end{array}$ & $\begin{array}{l}\text { IN4 as } \\
\text { comparable }\end{array}$ & $\begin{array}{l}\text { SGD as } \\
\text { comparable }\end{array}$ \\
\hline 6 & HTP & & & \\
\hline 7 & TPH & & & \\
\hline 8 & IN4 & $\begin{array}{l}\text { ECI as } \\
\text { comparable }\end{array}$ & $\begin{array}{l}\text { ECI as } \\
\text { comparable }\end{array}$ & $\begin{array}{l}\text { DAD as } \\
\text { comparable }\end{array}$ \\
\hline 9 & $\mathrm{ADC}$ & $\begin{array}{l}\text { DHI as } \\
\text { comparable }\end{array}$ & $\begin{array}{l}\text { HEV as } \\
\text { comparable }\end{array}$ & $\begin{array}{l}\text { DAE as } \\
\text { comparable }\end{array}$ \\
\hline 10 & HST & & & \\
\hline 11 & SGD & & & \\
\hline 12 & DAE & & & \\
\hline 13 & $\mathrm{HEV}$ & & & \\
\hline 14 & ALT & & & \\
\hline 15 & EFI & & & \\
\hline 16 & EID & & & \\
\hline 17 & DAD & & & \\
\hline 18 & SED & & & \\
\hline
\end{tabular}

\section{General Data Analysis}

The research sample has total 18 listed firms in the telecommunication and education industry market with the live data from the stock exchange. 
Firstly, we estimate equity beta values of these firms and use financial leverage to estimate asset beta values of them. Secondly, we change the competitors from what reported in F.S 2011 to those with size doubling and reducing slightly to see the sensitivity of beta values. We found out that in case leverage up 30\%, asset beta mean values increase if competitor size doubles and decrease if competitor size is smaller (correlated with the competitor size). Also in 3 scenarios of different leverage and current competitors, we find out equity beta mean values $(0,671,0,655$ and 0,682$)$ are moving in the opposite direction with the leverage. Leverage degree changes definitely has certain effects on asset and equity beta values.

\section{Empirical Research Findings and Discussion}

In the below section, data used are from total 18 listed telecommunication and education industry companies on VN stock exchange (HOSE and HNX mainly). In the scenario 1, current financial leverage degree is kept as in the 2011 financial statements which is used to calculate market risk (beta) whereas competitor size is kept as current, then changed from double size to slightly smaller size. Then, two (2) FL scenarios are changed up to $30 \%$ and down to $20 \%$, compared to the current FL degree. In short, the below table 1 shows three scenarios used for analyzing the risk level of these listed firms.

Market risk (beta) under the impact of tax rate, includes: 1) equity beta; and 2) asset beta.

Table 1 - Analyzing market risk under three (3) scenarios (Made by Author)

\begin{tabular}{|l|l|l|l|}
\hline $\begin{array}{l}\text { Competitor size as } \\
\text { current }\end{array}$ & FL as current & FL up 30\% & FL down 20\% \\
\hline $\begin{array}{l}\text { Competitor size } \\
\text { slightly smaller }\end{array}$ & & Scenario 2 & Scenario 3 \\
\cline { 1 - 1 } $\begin{array}{l}\text { Competitor size } \\
\text { double }\end{array}$ & & & \\
\hline
\end{tabular}

7.1 Scenario 1: current financial leverage (FL) as in financial reports 2011 and competitor size kept as current, slightly smaller and double

In this case, all beta values of 18 listed firms on $\mathrm{VN}$ telecommunication and education industry market as following: 
Table 2 - Market risk of listed companies on VN telecommunication and education industry market under a two factors model (case 1) (source: VN stock exchange 2012)

\begin{tabular}{|c|c|c|c|c|c|c|c|}
\hline \multirow[b]{2}{*}{$\begin{array}{l}\text { Order } \\
\text { No. } \\
\end{array}$} & \multirow[b]{2}{*}{$\begin{array}{l}\text { Company } \\
\text { stock code }\end{array}$} & \multirow{2}{*}{\begin{tabular}{|l}
$\begin{array}{l}\text { Competitor } \\
\text { size as } \\
\text { current }\end{array}$ \\
Equity beta \\
\end{tabular}} & \multirow{2}{*}{\begin{tabular}{|l|}
$\begin{array}{l}\text { Competitor } \\
\text { size slightly } \\
\text { smaller }\end{array}$ \\
Asset beta \\
(assume \\
debt beta = \\
()) \\
\end{tabular}} & \multirow{2}{*}{\begin{tabular}{|l}
$\begin{array}{l}\text { Competitor size } \\
\text { double }\end{array}$ \\
Equity beta \\
\end{tabular}} & & & \\
\hline & & & & & $\begin{array}{l}\text { Asset beta (assume } \\
\text { debt beta }=0 \text { ) }\end{array}$ & $\begin{array}{l}\text { Equity } \\
\text { beta }\end{array}$ & $\begin{array}{l}\text { Asset beta } \\
\text { (assume debt } \\
\text { beta }=0 \text { ) }\end{array}$ \\
\hline 1 & ECI & 0,708 & 0,527 & 0,708 & 0,527 & 0,708 & 0,527 \\
\hline 2 & INN & 0,195 & 0,104 & 0,195 & 0,104 & 0,195 & 0,104 \\
\hline 3 & PTP & $-0,524$ & $-0,251$ & $-0,524$ & $-0,251$ & $-0,524$ & $-0,251$ \\
\hline 4 & DHI & 0,740 & 0,547 & 0,740 & 0,547 & 0,740 & 0,547 \\
\hline 5 & IHK & 0,514 & 0,295 & 0,303 & 0,174 & 0,698 & 0,400 \\
\hline 6 & HTP & 1,091 & 0,846 & 1,091 & 0,846 & 1,091 & 0,846 \\
\hline 7 & $\mathrm{TPH}$ & 0,801 & 0,356 & 0,801 & 0,356 & 0,801 & 0,356 \\
\hline 8 & IN4 & 0,473 & 0,284 & 0,473 & 0,284 & 0,418 & 0,251 \\
\hline 9 & $\mathrm{ADC}$ & 0,425 & 0,214 & 0,363 & 0,183 & 0,400 & 0,201 \\
\hline 10 & HST & $-0,042$ & $-0,029$ & $-0,042$ & $-0,029$ & $-0,042$ & $-0,029$ \\
\hline 11 & SGD & 1,089 & 0,581 & 1,089 & 0,581 & 1,089 & 0,581 \\
\hline 12 & DAE & 0,696 & 0,275 & 0,696 & 0,275 & 0,696 & 0,275 \\
\hline 13 & $\mathrm{HEV}$ & 0,633 & 0,434 & 0,633 & 0,434 & 0,633 & 0,434 \\
\hline 14 & ALT & 0,759 & 0,607 & 0,759 & 0,607 & 0,759 & 0,607 \\
\hline 15 & EFI & 2,056 & 1,941 & 2,056 & 1,941 & 2,056 & 1,941 \\
\hline 16 & EID & 1,210 & 0,874 & 1,210 & 0,874 & 1,210 & 0,874 \\
\hline 17 & DAD & 0,625 & 0,423 & 0,625 & 0,423 & 0,625 & 0,423 \\
\hline 18 & SED & 0,634 & 0,292 & 0,634 & 0,292 & 0,634 & 0,292 \\
\hline
\end{tabular}

7.2. Scenario 2: financial leverage increases up to $30 \%$ and competitor size kept as current, slightly smaller and double

If leverage increases up to $30 \%$, all beta values of total 18 listed firms on VN telecommunication and education industry market as below:

Table 3 - Market risks of listed telecommunication and education industry firms under a two factors model (case 2) (source: VN stock exchange 2012)

\begin{tabular}{|c|c|c|c|c|c|c|c|}
\hline \multirow[b]{2}{*}{$\begin{array}{l}\text { Order } \\
\text { No. }\end{array}$} & \multirow[b]{2}{*}{$\begin{array}{l}\text { Company } \\
\text { stock code }\end{array}$} & \multicolumn{2}{|c|}{ Competitor size as current } & \multicolumn{2}{|c|}{$\begin{array}{l}\text { Competitor size slightly } \\
\text { smaller }\end{array}$} & \multicolumn{2}{|c|}{ Competitor size double } \\
\hline & & $\begin{array}{l}\text { Equity } \\
\text { beta }\end{array}$ & $\begin{array}{l}\text { Asset beta (assume } \\
\text { debt beta = } 0 \text { ) }\end{array}$ & $\begin{array}{l}\text { Equity } \\
\text { beta }\end{array}$ & $\begin{array}{l}\text { Asset beta } \\
\text { (assume debt } \\
\text { beta = 0) }\end{array}$ & $\begin{array}{l}\text { Equity } \\
\text { beta }\end{array}$ & $\begin{array}{l}\text { Asset beta } \\
\text { (assume } \\
\text { debt } \\
\text { beta = 0) }\end{array}$ \\
\hline 1 & ECI & 0,708 & 0,473 & 0,708 & 0,473 & 0,708 & 0,473 \\
\hline 2 & INN & 0,195 & 0,077 & 0,195 & 0,077 & 0,195 & 0,077 \\
\hline 3 & PTP & $-0,524$ & $-0,169$ & $-0,524$ & $-0,169$ & $-0,524$ & $-0,169$ \\
\hline
\end{tabular}




\begin{tabular}{|l|l|l|l|l|l|l|l|}
\hline 4 & DHI & 0,740 & 0,489 & 0,740 & 0,489 & 0,740 & 0,489 \\
\hline 5 & IHK & 0,414 & 0,184 & 0,202 & 0,090 & 0,563 & 0,250 \\
\hline 6 & HTP & 1,091 & 0,772 & 1,091 & 0,772 & 1,091 & 0,772 \\
\hline 7 & TPH & 0,801 & 0,223 & 0,801 & 0,223 & 0,801 & 0,223 \\
\hline 8 & IN4 & 0,392 & 0,189 & 0,392 & 0,189 & 0,346 & 0,166 \\
\hline 9 & ADC & 0,312 & 0,110 & 0,267 & 0,094 & 0,294 & 0,104 \\
\hline 10 & HST & $-0,042$ & $-0,025$ & $-0,042$ & $-0,025$ & $-0,042$ & $-0,025$ \\
\hline 11 & SGD & 1,089 & 0,429 & 1,089 & 0,429 & 1,089 & 0,429 \\
\hline 12 & DAE & 0,696 & 0,148 & 0,696 & 0,148 & 0,696 & 0,148 \\
\hline 13 & HEV & 0,633 & 0,374 & 0,633 & 0,374 & 0,633 & 0,374 \\
\hline 14 & ALT & 0,759 & 0,561 & 0,759 & 0,561 & 0,759 & 0,561 \\
\hline 15 & EFI & 2,056 & 1,906 & 2,056 & 1,906 & 2,056 & 1,906 \\
\hline 16 & EID & 1,210 & 0,773 & 1,210 & 0,773 & 1,210 & 0,773 \\
\hline 17 & DAD & 0,625 & 0,363 & 0,625 & 0,363 & 0,625 & 0,363 \\
\hline 18 & SED & 0,634 & 0,189 & 0,634 & 0,189 & 0,634 & 0,189 \\
\hline
\end{tabular}

7.3. Scenario 3: leverage decreases down to $20 \%$ and competitor size kept as current, slightly smaller and double

If leverage decreases down to $20 \%$, all beta values of total 18 listed firms on the telecommunication and education industry market in $\mathrm{VN}$ as following:

Table 4 - Market risk of listed telecommunication and education industry firms under a two factors model (case 3) (source: VN stock exchange 2012)

\begin{tabular}{|c|c|c|c|c|c|c|c|}
\hline \multirow[b]{2}{*}{$\begin{array}{l}\text { Order } \\
\text { No. }\end{array}$} & \multirow[b]{2}{*}{$\begin{array}{l}\text { Company } \\
\text { stock code }\end{array}$} & \multicolumn{2}{|c|}{$\begin{array}{l}\text { Competitor size as } \\
\text { current }\end{array}$} & \multicolumn{2}{|c|}{$\begin{array}{l}\text { Competitor size slightly } \\
\text { smaller }\end{array}$} & \multicolumn{2}{|c|}{ Competitor size double } \\
\hline & & $\begin{array}{l}\text { Equity } \\
\text { beta }\end{array}$ & $\begin{array}{l}\text { Asset beta } \\
\text { (assume debt } \\
\text { beta }=0)\end{array}$ & $\begin{array}{l}\text { Equity } \\
\text { beta }\end{array}$ & $\begin{array}{l}\text { Asset beta } \\
(\text { assume debt } \\
\text { beta }=0)\end{array}$ & Equity beta & $\begin{array}{l}\text { Asset beta } \\
\text { (assume debt } \\
\text { beta }=0)\end{array}$ \\
\hline 1 & ECI & 0,708 & 0,564 & 0,708 & 0,564 & 0,708 & 0,564 \\
\hline 2 & INN & 0,195 & 0,123 & 0,195 & 0,123 & 0,195 & 0,123 \\
\hline 3 & PTP & $-0,524$ & $-0,305$ & $-0,524$ & $-0,305$ & $-0,524$ & $-0,305$ \\
\hline 4 & DHI & 0,740 & 0,586 & 0,740 & 0,586 & 0,740 & 0,586 \\
\hline 5 & IHK & 0,577 & 0,380 & 0,377 & 0,248 & 0,784 & 0,516 \\
\hline 6 & HTP & 1,091 & 0,895 & 1,091 & 0,895 & 1,091 & 0,895 \\
\hline 7 & TPH & 0,801 & 0,445 & 0,801 & 0,445 & 0,801 & 0,445 \\
\hline 8 & IN4 & 0,524 & 0,357 & 0,524 & 0,357 & 0,463 & 0,315 \\
\hline 9 & $\mathrm{ADC}$ & 0,495 & 0,298 & 0,423 & 0,255 & 0,466 & 0,280 \\
\hline 10 & HST & $-0,042$ & $-0,032$ & $-0,042$ & $-0,032$ & $-0,042$ & $-0,032$ \\
\hline 11 & SGD & 1,089 & 0,683 & 1,089 & 0,683 & 1,089 & 0,683 \\
\hline 12 & DAE & 0,696 & 0,359 & 0,696 & 0,359 & 0,696 & 0,359 \\
\hline 13 & $\mathrm{HEV}$ & 0,633 & 0,474 & 0,633 & 0,474 & 0,633 & 0,474 \\
\hline 14 & ALT & 0,759 & 0,637 & 0,759 & 0,637 & 0,759 & 0,637 \\
\hline 15 & EFI & 2,056 & 1,964 & 2,056 & 1,964 & 2,056 & 1,964 \\
\hline
\end{tabular}




\begin{tabular}{|l|l|l|l|l|l|l|l|}
\hline 16 & EID & 1,210 & 0,941 & 1,210 & 0,941 & 1,210 & 0,941 \\
\hline 17 & DAD & 0,625 & 0,464 & 0,625 & 0,464 & 0,625 & 0,464 \\
\hline 18 & SED & 0,634 & 0,360 & 0,634 & 0,360 & 0,634 & 0,360 \\
\hline
\end{tabular}

All three above tables and data show that values of equity and asset beta in the case of increasing leverage up to $30 \%$ or decreasing leverage degree down to $20 \%$ have certain fluctuation.

\section{Comparing statistical results in $\mathbf{3}$ scenarios of changing leverage:}

Table 5 - Statistical results (FL in case 1) (source: VN stock exchange 2012)

\begin{tabular}{|c|c|c|c|c|c|c|c|c|c|}
\hline \multirow[b]{2}{*}{$\begin{array}{l}\text { Statistic } \\
\text { results }\end{array}$} & \multicolumn{2}{|c|}{$\begin{array}{l}\text { Competitor size as } \\
\text { current }\end{array}$} & & \multicolumn{2}{|c|}{\begin{tabular}{|lr}
$\begin{array}{l}\text { Competitor } \\
\text { slightly smaller }\end{array}$ & size \\
\end{tabular}} & & \multicolumn{2}{|c|}{\begin{tabular}{|l|}
$\begin{array}{l}\text { Competitor size } \\
\text { double }\end{array}$ \\
\end{tabular}} & \\
\hline & $\begin{array}{l}\text { Equity } \\
\text { beta }\end{array}$ & $\begin{array}{l}\text { Asset beta } \\
\text { (assume } \\
\text { debt } \\
\text { beta }=0 \text { ) }\end{array}$ & Difference & $\begin{array}{l}\text { Equity } \\
\text { beta }\end{array}$ & $\begin{array}{l}\text { Asset beta } \\
\text { (assume debt } \\
\text { beta }=0 \text { ) }\end{array}$ & Difference & $\begin{array}{l}\text { Equity } \\
\text { beta }\end{array}$ & \begin{tabular}{|l} 
Asset \\
beta \\
(assume \\
debt \\
beta $=0$ ) \\
\end{tabular} & Difference \\
\hline MAX & 2,056 & 1,941 & 0,115 & 2,056 & 1,941 & 0,115 & 2,056 & 1,941 & 0,115 \\
\hline MIN & $-0,524$ & $-0,251$ & $-0,273$ & $-0,524$ & $-0,251$ & $-0,273$ & $-0,524$ & $-0,251$ & $-0,273$ \\
\hline MEAN & 0,671 & 0,462 & 0,209 & 0,656 & 0,454 & 0,202 & 0,677 & 0,466 & 0,212 \\
\hline VAR & 0,2872 & 0,2133 & 0,074 & 0,2955 & 0,2174 & 0,078 & 0,2880 & 0,2130 & 0,075 \\
\hline
\end{tabular}

Table 6 - Statistical results (FL in case 2) (source: VN stock exchange 2012)

\begin{tabular}{|c|c|c|c|c|c|c|c|c|c|}
\hline & $\begin{array}{l}\text { Compe } \\
\text { current }\end{array}$ & itor size as & & $\begin{array}{l}\text { Comp } \\
\text { slightly }\end{array}$ & $\begin{array}{ll}\text { titor } & \text { size } \\
\text { smaller }\end{array}$ & & $\begin{array}{l}\text { Compe } \\
\text { double }\end{array}$ & titor size & \\
\hline $\begin{array}{l}\text { Statistic } \\
\text { results }\end{array}$ & $\begin{array}{l}\text { Equity } \\
\text { beta }\end{array}$ & \begin{tabular}{|l|} 
Asset \\
beta \\
(assume \\
debt \\
beta = 0) \\
\end{tabular} & Difference & $\begin{array}{l}\text { Equity } \\
\text { beta }\end{array}$ & $\begin{array}{l}\text { Asset beta } \\
\text { (assume } \\
\text { debt } \\
\text { beta =0) }\end{array}$ & Difference & $\begin{array}{l}\text { Equity } \\
\text { beta }\end{array}$ & $\begin{array}{l}\text { Asset } \\
\text { beta } \\
\text { (assume } \\
\text { debt } \\
\text { beta = 0) }\end{array}$ & Difference \\
\hline MAX & 2,056 & 1,906 & 0,149 & 2,056 & 1,906 & 0,149 & 2,056 & 1,906 & 0,149 \\
\hline MIN & $-0,524$ & $-0,169$ & $-0,355$ & $-0,524$ & $-0,169$ & $-0,355$ & $-0,524$ & $-0,169$ & $-0,355$ \\
\hline MEAN & 0,655 & 0,393 & 0,262 & 0,641 & 0,386 & 0,254 & 0,660 & 0,386 & 0,273 \\
\hline VAR & 0,2956 & 0,2065 & 0,089 & 0,3060 & 0,2099 & 0,096 & 0,2950 & 0,2059 & 0,089 \\
\hline
\end{tabular}

Table 7- Statistical results (FL in case 3) (source: VN stock exchange 2012)

\begin{tabular}{|c|c|c|c|c|c|c|c|c|c|}
\hline \multirow[b]{2}{*}{$\begin{array}{l}\text { Statistic } \\
\text { results }\end{array}$} & \multicolumn{2}{|c|}{$\begin{array}{l}\text { Competitor size as } \\
\text { current }\end{array}$} & & \multicolumn{2}{|c|}{$\begin{array}{l}\text { Competitor size } \\
\text { slightly smaller }\end{array}$} & & \multicolumn{2}{|c|}{$\begin{array}{l}\text { Competitor size } \\
\text { double }\end{array}$} & \\
\hline & $\begin{array}{l}\text { Equity } \\
\text { beta }\end{array}$ & $\begin{array}{l}\text { Asset } \\
\text { beta } \\
\text { (assume } \\
\text { debt } \\
\text { beta = 0) }\end{array}$ & Difference & $\begin{array}{l}\text { Equity } \\
\text { beta }\end{array}$ & $\begin{array}{l}\text { Asset beta } \\
\text { (assume debt } \\
\text { beta = } 0 \text { ) }\end{array}$ & Difference & $\begin{array}{l}\text { Equity } \\
\text { beta }\end{array}$ & $\begin{array}{l}\text { Asset } \\
\text { beta } \\
\text { (assume } \\
\text { debt } \\
\text { beta }=0 \text { ) }\end{array}$ & Difference \\
\hline MAX & 2,056 & 1,964 & 0,092 & 2,056 & 2,056 & 0,000 & 2,056 & 1,964 & 0,092 \\
\hline MIN & $-0,524$ & $-0,305$ & $-0,219$ & $-0,524$ & $-0,524$ & 0,000 & $-0,524$ & $-0,305$ & $-0,219$ \\
\hline
\end{tabular}




\begin{tabular}{|l|l|l|l|l|l|l|l|l|l|}
\hline MEAN & 0,682 & 0,511 & 0,171 & 0,598 & 0,490 & 0,108 & 0,667 & 0,454 & 0,213 \\
\hline VAR & 0,2834 & 0,2205 & 0,063 & 0,2945 & 0,2839 & 0,011 & 0,2898 & 0,2246 & 0,065 \\
\hline
\end{tabular}

Based on the calculated results, we find out:

First of all, if competitor size is kept as current, both max and min values of asset beta vary in 3 cases (max values of asset beta decreases to 1,906 and increases to 1,964 when leverage up $30 \%$ and down $20 \%$ ). Secondly, if competitor size is chosen with total asset doubling, max values of asset beta vary in all 3 scenarios. Thirdly, if competitor is chosen with total asset slightly smaller, there is no changes in min values of equity whereas asset beta min values decreases to $-0,524$ if leverage down $20 \%$ and increases to $-0,169$ if leverage up $30 \%$.

Furthermore, the below chart 1 shows us : in the case competitor size doubles, the risk is less dispersed if leverage up to $30 \%$. Especially, equity beta var reduces to 0,295 . On the contrary, in the case of slightly smaller size competitors, if leverage up to $30 \%$, equity beta var increases to 0,641 and the risk is more dispersed.

Last but not least, from chart 2 , we could note that in the case of slightly smaller size competitors, keeping the current leverage degree, asset beta mean value reduces to 0,454 . On the other hand, in the case of doubling size competitors, asset beta mean value goes up to 0,466 .

Chart 1 - Comparing statistical results of equity beta var and mean in three (3) scenarios of changing FL and competitor size (source: VN stock exchange 2012)

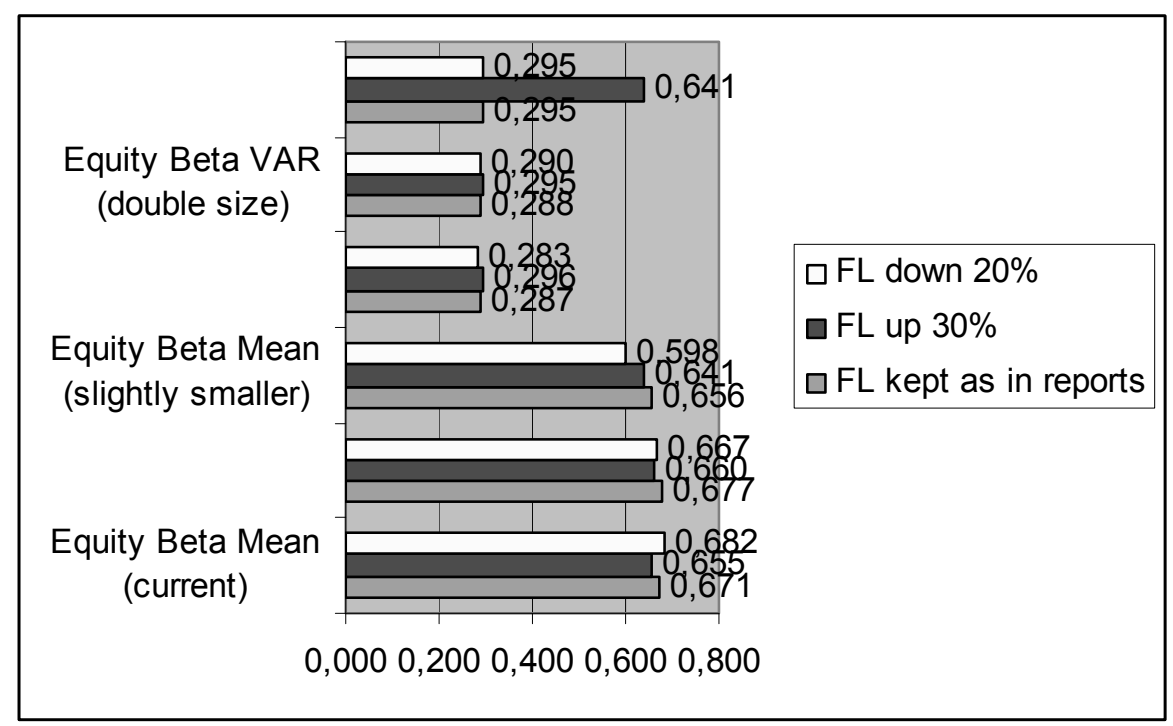


Chart 2 - Comparing statistical results of asset beta var and mean in three (3) scenarios of changing FL and competitor size (source: VN stock exchange 2012)

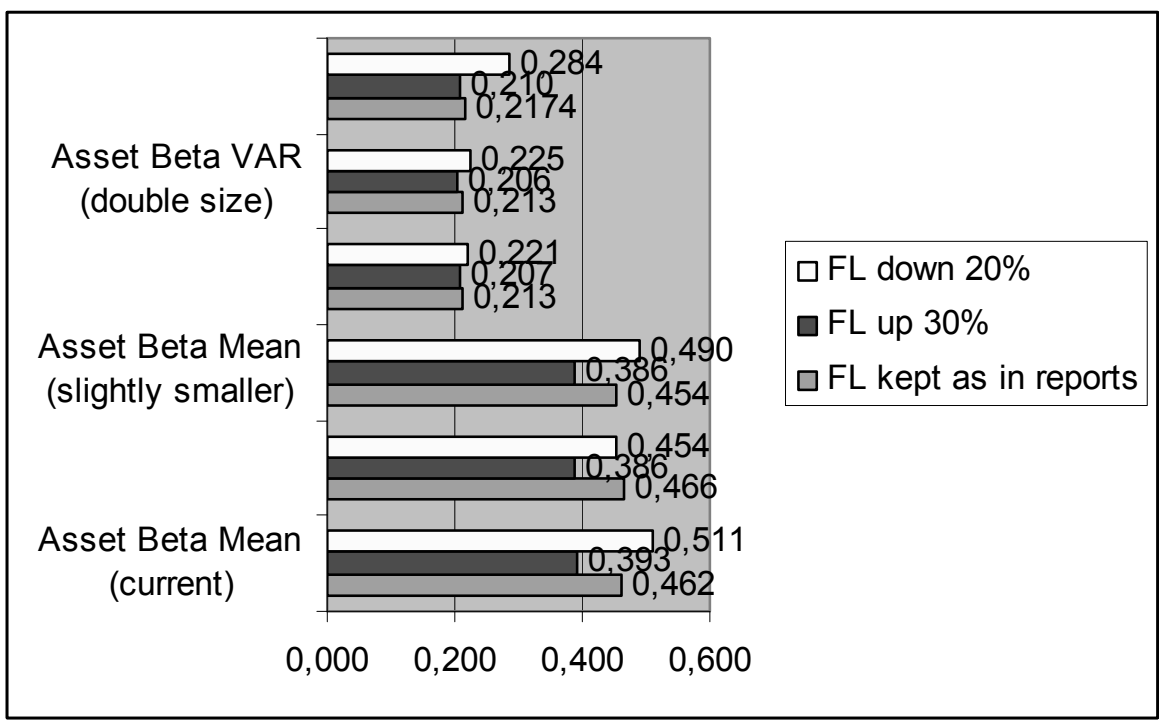

\section{Conclusion and Policy suggestion}

In general, the government has to consider the impacts on the mobility of capital in the markets when it changes the macro policies and the legal system and regulation for developing the telecommunication and education market. The Ministry of Finance continues to increase the effectiveness of fiscal policies and tax policies which are needed to combine with other macro policies at the same time. The State Bank of Viet Nam continues to increase the effectiveness of capital providing channels for telecommunication and education companies. Furthermore, the entire efforts among many different government bodies need to be coordinated.

Last but not least, these companies might be aware of a minimum value of asset beta mean of 0,386 with either smaller or doubling size competitors (leverage up $30 \%$ ) and a maximum value of asset beta mean of 0,511 with approximate size competitors and leverage down $20 \%$. The riskier the marketing strategy, the lower the market risk.

Finally, this paper suggests implications for further research and policy suggestion for the Viet Nam government and relevant organizations, economists and investors from current market conditions.

\section{References}

1. Carvajal, Santiago G., Sanabria, Pedro., and Ospina, Daniel., (2013), Balanced Scorecard for Entrepreneurial Strategic Marketing in Comlumbia, Global Journal of Business Research 
2. Esposito, Annamaria., (2013), Insights about Integrated Marketing Communication in Small And Medium-Sized Italian Enterprises, Business System Review, Vol.2, Issue 1

3. Flifel, Kaouther., (2012), Financial Markets between Efficiency and Persistence : Empirical Evidence on Daily Data, Asian Journal of Finance and Accounting

4. Gao, Huasheng., Harford, Jarrad., and Li, Kai., (2013), Determinants of Corporate Cash Policy: Insights from Private Firms, Journal of Financial Economics

5. Huy, Dinh T.N., (2012), Estimating Beta of Viet Nam listed construction companies groups during the crisis, Journal of Integration and Development

6. Kale, Jayant R., Meneghetti, Costanza., and Sharur, Husayn., (2013), Contracting With Non-Financial Stakeholders and Corporate Capital Structure: The Case of Product Warantties, Journal of Financial and Quantitative Analysis

7. Litvak, Kate., (2008), Defensive Management: Does the Sarbanes-Oxley Act Discourage Corporate Risk-Taking?, Law and Economics Research Paper, No. 108

8. Ling, Amy., (2013), Tax Issues Relating to Intangibles, Asia-Pacific Tax Bulletin

9. Lu, Wenling., and Whidbee, David A., (2013), Bank Structure and Failure,Journal of Financial Econoic Policy

10. XiYing Zhang, Ivy., (2007),Economic consequences of the Sarbanes-Oxley Act of 2002, Journal of Accounting and Economics, 44 (2007) 74-115

\section{Research}

1. Ang, A., Chen, J., (2007), CAPM Over the Long Run: 1926-2001, Journal of Empirical Finance

2. Baker, Kent H., Singleton, Clay J., and Veit, Theodore E., (2011), Survey Research in Corporate Finance: Bridging The Gap Between Theory and Practice, Oxford University Press

3. ADB and Viet Nam Fact Sheet, 2010

\section{Other web sources}

http://www.mofa.gov.vn/vi/

http://www.hsx.vn/hsx/

www.tuoitre.com.vn;

www.saigontimes.com.vn; 


\section{Exhibit}

Exhibit 1- VNI Index and other stock market index during crisis 2006-2010 (source: global stock exchange 2012)

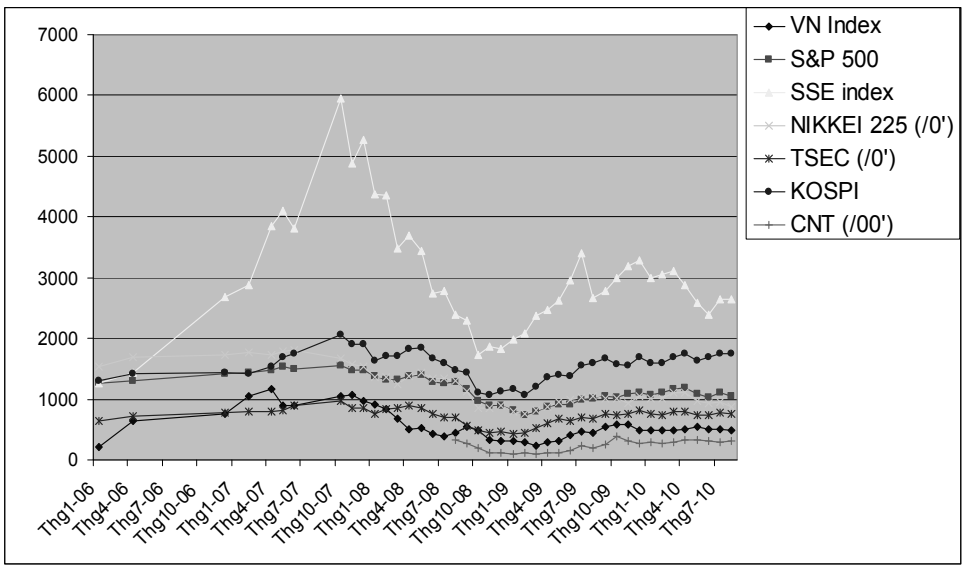

Exhibit 2 - Inflation, GDP growth and macroeconomics factors (source: Viet Nam commercial banks and economic statistical bureau)

$\begin{array}{lccl}\text { Year } & \text { Inflation } & \text { GDP } & \text { USD/VND rate } \\ 2011 & 18 \% & 5,89 \% & 20.670 \\ 2010 & 11,75 \% & 6,5 \% & 19.495 \\ & \begin{array}{c}\text { (Estimated at Dec } \\ \text { (expected) }\end{array} \\ 2009 & 6,88 \% & 5,2 \% & \\ 2008 & 22 \% & 6,23 \% & 17.000 \\ 2007 & 12,63 \% & 8,44 \% & 17.700 \\ 2006 & 6,6 \% & 8,17 \% & \\ 2005 & 8,4 \% & & \\ \text { Note } & \text { approximately } & & \end{array}$

Exhibit 3 - Financial leverage degree of listed telecommunication and education firms in three (3) scenarios with different competitors

(source: Viet Nam commercial banks and economic statistical bureau) 


\begin{tabular}{|l|l|l|l|l|}
\hline $\begin{array}{l}\text { Order } \\
\text { No. }\end{array}$ & $\begin{array}{l}\text { Company } \\
\text { Stock } \\
\text { code }\end{array}$ & $\begin{array}{l}\text { FL as } \\
\text { current }\end{array}$ & $\begin{array}{l}\text { FL up } \\
30 \%\end{array}$ & $\begin{array}{l}\text { FL } \\
\text { down } \\
20 \%\end{array}$ \\
\hline 1 & ECI & $25,5 \%$ & $33,2 \%$ & $20,4 \%$ \\
\hline 2 & INN & $46,6 \%$ & $60,5 \%$ & $37,2 \%$ \\
\hline 3 & PTP & $52,1 \%$ & $67,8 \%$ & $41,7 \%$ \\
\hline 4 & DHI & $26,1 \%$ & $33,9 \%$ & $20,9 \%$ \\
\hline 5 & IHK & $42,7 \%$ & $55,5 \%$ & $34,2 \%$ \\
\hline 6 & HTP & $22,5 \%$ & $29,2 \%$ & $18,0 \%$ \\
\hline 7 & TPH & $55,5 \%$ & $72,2 \%$ & $44,4 \%$ \\
\hline 8 & IN4 & $39,9 \%$ & $51,9 \%$ & $31,9 \%$ \\
\hline 9 & ADC & $49,7 \%$ & $64,7 \%$ & $39,8 \%$ \\
\hline 10 & HST & $30,4 \%$ & $39,6 \%$ & $24,4 \%$ \\
\hline 11 & SGD & $46,6 \%$ & $60,6 \%$ & $37,3 \%$ \\
\hline 12 & DAE & $60,6 \%$ & $78,7 \%$ & $48,5 \%$ \\
\hline 13 & HEV & $31,4 \%$ & $40,9 \%$ & $25,2 \%$ \\
\hline 14 & ALT & $20,1 \%$ & $26,1 \%$ & $16,1 \%$ \\
\hline 15 & EFI & $5,6 \%$ & $7,3 \%$ & $4,5 \%$ \\
\hline 16 & EID & $27,8 \%$ & $36,2 \%$ & $22,2 \%$ \\
\hline 17 & DAD & $32,3 \%$ & $42,0 \%$ & $25,9 \%$ \\
\hline 18 & SED & $53,9 \%$ & $70,1 \%$ & $43,1 \%$ \\
\hline & Average & $37,2 \%$ & $48,4 \%$ & $29,8 \%$ \\
\cline { 2 - 5 } & & & & \\
\hline & & & $\% \%$ & $\%$ \\
\hline
\end{tabular}

\section{Acknowledgements}

I would like to take this opportunity to express my warm thanks to Board of Editors and Colleagues at Citibank-HCMC, SCB and BIDV-HCMC, Dr. Chen and Dr. Yu Hai-Chin at Chung Yuan Christian University for class lectures, also Dr Chet Borucki, Dr Jay and my ex-Corporate Governance sensei, Dr. Shingo Takahashi at International University of Japan. My sincere thanks are for the editorial office, for their work during my research. Also, my warm thanks are for Dr. Ngo Huong, Dr. Ho Dieu, Dr. Ly H. Anh, Dr Nguyen V. Phuc and my lecturers at Banking University - HCMC, Viet Nam for their help.

Lastly, thank you very much for my family, colleagues, and brother in assisting convenient conditions for my research paper. 